\title{
Neural correlates of cough hypersensitivity in humans: evidence for central sensitisation and dysfunctional inhibitory control
}

\author{
Ayaka Ando, ${ }^{1,2}$ David Smallwood, ${ }^{3}$ Marcus McMahon, ${ }^{4}$ Louis Irving, ${ }^{3}$ \\ Stuart B Mazzone, ${ }^{1}$ Michael J Farrell ${ }^{5}$
}

- Additional material is published online only. To view please visit the journal online (http://dx.doi.org/10.1136/ thoraxjnl-2015-207425)

${ }^{1}$ School of Biomedical Sciences, The University of Queensland, St Lucia, Queensland, Australia

${ }^{2}$ The Florey Institute of Neuroscience and Mental Health, University of Melbourne, Parkville, Victoria, Australia

${ }^{3}$ Department of Respiratory and Sleep Medicine, Royal Melbourne Hospital, Parkville, Victoria, Australia

${ }^{4}$ Department of Respiratory and Sleep Medicine, Austin Hospital, Heidelberg, Victoria, Australia

${ }^{5}$ Department of Medical Imaging and Radiation Sciences, Monash University, Clayton, Victoria, Australia

Correspondence to Associate Professor Stuart B Mazzone, School of Biomedical Sciences, The University of Queensland, St Lucia, QLD 4072, Australia; s.mazzone@ uq.edu.au

Received 15 June 2015 Revised 3 January 2016 Accepted 7 January 2016 Published Online First 9 February 2016

\section{ABSTRACT}

Introduction Chronic non-productive coughing is a major complication of pulmonary disease and can also occur in many individuals without identifiable underlying pathology. The common clinical link in patients with cough is an enhanced sensitivity of the respiratory system to stimuli that subsequently evoke excessive coughing. The aetiology of this 'cough hypersensitivity syndrome' is unclear but believed to involve hypersensitivity of the sensory neural pathways that innervate the airways and lungs.

Methods In the present study, we used functional brain imaging to compare central neural responses to airway stimulation using inhaled capsaicin in healthy people and patients with cough hypersensitivity. Results Hypersensitivity in response to inhaled capsaicin coincided with elevated neural activity in the midbrain in a region encompassing the nucleus cuneiformis (left: $p<0.001$; right: $p<0.001$ ) and periaqueductal gray $(p=0.008)$ in comparison to normal sensitivity in controls. The enhanced activity noted in the midbrain is similar to that occurring in patients with chronic pain, thus providing empirical evidence to support the notion that cough and pain share neurobiological similarities. Furthermore, patients with cough hypersensitivity displayed difficulty controlling their cough, which manifested as a failure to suppress cough during capsaicin challenge (ie, reduced cough frequency) in controls compared with patients with cough hypersensitivity $(p=0.046)$. Cough suppression was associated with reduced activity in a forebrain network that included the dorsomedial prefrontal and anterior mid-cingulate cortices. Additionally, cough frequency was correlated with activity in the right inferior frontal gyrus $\left(R^{2}=0.6, p<0.001\right)$ and right anterior insula $\left(R^{2}=0.6\right.$, $\mathrm{p}<0.001)$, regions previously implicated in voluntary cough suppression.

Conclusions These findings provide insight into the central neurobiology of cough hypersensitivity and suggest that both central amplification of cough sensory inputs and reduced capacity to suppress cough motor behaviours define patients with problematic cough.

\section{INTRODUCTION}

Inhalation or aspiration of a noxious substance induces a perceivable sense of irritation, described as an 'urge to cough', indicative of neural pathways arising from the airways and projecting to higher brain centres that encode sensory perception. ${ }^{1} 2$

\section{Key messages}

\section{What is the key question?}

- Cough hypersensitivity is a common comorbidity of pulmonary disease and we asked the difficult question, what is the brain's involvement in cough hypersensitivity and chronic cough?

\section{What is the bottom line?}

- Using functional brain imaging, patients with cough hypersensitivity showed activation in the midbrain during airways irritation that does not occur in healthy people, whereas healthy people showed activation in the medial prefrontal cortex that is absent in the patients.

\section{Why read on?}

- Midbrain activity appears in hyperalgesic pain states, which suggests a common mechanism for increased pain and cough sensitivity, while decreased medial prefrontal responses in patients indicates a failure of this key component of the cough-suppression network.

Noxious airway sensations provide conscious feedback to the individual about the pulmonary environment, motivating behavioural respiratory modifications (such as coughing) to limit further exposure and help clear the airways of the offending irritant. ${ }^{3}$ Thus, the urge to cough represents an important component of airway defence against internal and external irritants, ensuring the maintenance of airway patency.

While in healthy individuals, the sensorimotor dimensions of cough are essential for airway protection, patients with some pulmonary diseases exhibit hypersensitivity to respiratory stimuli, ${ }^{3}$ leading to exaggerated coughing. Chronic cough is a debilitating condition representing a common reason for people to seek medical advice. In these patients, the urge to cough and cough are no longer protective as the hypersensitivity drives exaggerated perceptions of irritation, such that innocuous activities (including laughing or talking) are sufficient to induce a bout of severe coughing. ${ }^{3-5}$ This patient phenotype characterises 'cough hypersensitivity syndrome', 6 a challenging condition for respiratory physicians to
Smallwood D, McMahon $M_{1}$ 323-329. 
treat as available therapeutics provide inadequate relief largely due to a lack of understanding of the basic mechanisms evoking the hypersensitive state.

Cough hypersensitivity syndrome shares similarities with chronic pain, characterised by hyperalgesia (exaggerated pain to noxious stimuli) and allodynia (pain in response to non-painful stimuli), akin to the hypertussive and allotussive states described in patients with cough. ${ }^{3}$ Cough, like pain, occurs across a wide range of diseases, and can be present in many patients without any identifiable pathology. ${ }^{8} 9$ This argues for convergence of disparate disease processes onto common mechanisms driving cough hypersensitivity $(\mathrm{CH})$, which could be exploited for therapeutic control. It also argues for mutually informative mechanisms contributing to cough and pain. Indeed, the neural networks governing unpleasant airway and somatic sensations are remarkably similar ${ }^{10}$ and compounds used to treat neuropathic pain (eg, gabapentin) also have efficacy in patients with cough. $^{11}$

Chronic pain is accompanied by sensitisation of peripheral sensory neurons as well as changes to neural processing in central nervous system (CNS) areas important for integrating sensory inputs. ${ }^{12-14}$ Likewise, cough hypersensitivity may involve peripheral and central components. Animal studies have described how airways inflammation impinges on the primary respiratory sensory neurons that provide the initial encoding of cough, ${ }^{15}$ but the CNS contribution to cough hypersensitivity is unknown. In healthy humans, functional brain imaging has revealed the central neural correlates of the urge to cough, cough and cough suppression, ${ }^{16-20}$ providing a priori predictions for how cough hypersensitivity may develop. ${ }^{1}$ In the present study, we directly assess this by comparing regional brain responses to airways irritation using functional brain imaging in healthy participants and patients with cough hypersensitivity. We hypothesise that patients with cough hypersensitivity would have altered functional responses in brain regions encoding the urge to cough and/or voluntary cough suppression during capsaicin inhalation. ${ }^{16} 19$

\section{MATERIALS AND METHODS}

\section{Recruitment and experimental procedures}

Participants provided consent to be involved in the study according to procedures approved by the Melbourne Health Human Research Ethics Committee (approval \#2010.085). Patients with $\mathrm{CH}$ were recruited from two Respiratory Medicine Units at tertiary healthcare centres in Melbourne, Australia. The patients with $\mathrm{CH}$ were defined as people suffering from persistent cough for at least 8 weeks with no upper respiratory tract infection in the same preceding period. Furthermore, these patients scored 14 or more on the Hull Airway Reflux Questionnaire (HARQ), which was a questionnaire originally developed to identify airway reflux but has recently been demonstrated to have good construct and criterion validity as a diagnostic instrument for cough hypersensitivity syndrome. ${ }^{21}$ Healthy controls were recruited according to demographic attributes to permit age and sex matching with individual patients with $\mathrm{CH}$. None of the participants had a clinical diagnosis of anxiety or panic disorders.

All patients with $\mathrm{CH}$ shared a common symptom of chronic cough that lasted for mean of $4.6 \pm 6.5$ years, ranging from 8 weeks to 20 years. Most participants were refractory cough sufferers where various treatments had previously been unsuccessful. However, three patients with $\mathrm{CH}$ were on oesophageal reflux medication and one patient with $\mathrm{CH}$ was on medication for allergic rhinitis and COPD. Although the patients with $\mathrm{CH}$ did not have a uniform underlying disease, they all suffered from a common symptom of chronic cough, which was the key characteristic of interest in this study.

During the psychophysical testing session, the method of limits of was used to determine capsaicin sensitivity. All participants inhaled a single vital capacity of nebulised capsaicin that was prepared in doubling doses $(0.06-125 \mu \mathrm{M})$ and rated urge to cough on an 11-point numerical rating scale $(0=$ no urge to cough, $10=$ maximum urge to cough). Cough frequency was recorded after inhalations. The lowest dose at which a non-zero rating of urge to cough occurred was deemed the $\mathrm{Cu}$ threshold, and the lowest dose to evoke two or more coughs was the $\mathrm{C} 2$ threshold. The highest dose that could be inhaled repetitively for $24 \mathrm{~s}$ without a coughing event was defined as the maximum suppressible $\left(S_{\max }\right)$ dose and this dose was used in the subsequent scanning session. After $\mathrm{Cu}, \mathrm{C} 2$ and $\mathrm{S}_{\max }$ concentrations were determined, a further 10 stimuli $(2 \times 5$ doses $)$ were delivered in randomised order to generate a stimulus response function. The stimulus intensities were two stimuli each at (i) the intensity corresponding with the $\mathrm{C} 2$ concentration, (ii) one dose below and above the $\mathrm{C} 2(\mathrm{C} 2 \pm 1)$ and (iii) two doses below and above the $\mathrm{C} 2(\mathrm{C} 2 \pm 2)$.

During the image acquisition session, participants lay comfortably on the scanner bed with their head stabilised with foam padding. Participants were also fitted with the facemask and nebulising apparatus used in the psychophysical testing session with the replacement of the air pump with medical air (flow rate $=0.7 \mathrm{~mL} / \mathrm{min}$ ). A periscope mirror attached to the scanner head coil enabled participants to view a projector screen that provided visual cues throughout the experimental session.

The imaging protocol included eight blocks of $24 \mathrm{~s}$ periods where the participants were administered either saline, a low or a high dose of capsaicin in a randomised order interspersed by $42 \mathrm{~s}$ of no-stimulation periods. Participants were asked to rate the level of urge to cough after each capsaicin challenge and the number of coughs was also noted.

All participants were administered two different capsaicin concentrations during this session, where one was their $S_{\max }$ dose (high dose) and another lower capsaicin dose (low dose). The low dose was determined differently in the patients with $\mathrm{CH}$ and healthy controls. Low dose for patients with $\mathrm{CH}$ were two capsaicin doses below their $S_{\max }$ dose whereas the control group received the $S_{\max }$ dose of their matched $\mathrm{CH}$ participant. However, not all control participants had a higher capsaicin threshold than their matched $\mathrm{CH}$ participant. In two cases, the participant in the healthy control group was administered the low dose of the individually matched patient with $\mathrm{CH}$.

\section{Image acquisition}

Scanning was performed at the Murdoch Children's Research Institute (Melbourne, Australia) using a Siemens Trio $3 \mathrm{~T}$ scanner (Siemens) with a 32 channel head coil. Structural T1-weighted images were acquired in the sagittal plane (192 slices; $0.90 \mathrm{~mm}$ thickness; $0.84 \times 0.84 \mathrm{~mm}^{2}$ in-plane resolution; echo time (TE) $2.6 \mathrm{~ms}$; repetition time (TR) $1900 \mathrm{~ms}$; flip angle $\left.9^{\circ}\right)$. Echo-planar images (EPI) were acquired in the transaxial plane (36 slices; $4 \mathrm{~mm}$ thickness; $3.3 \times 3.3 \mathrm{~mm}^{2}$ in-plane resolution; TE $35 \mathrm{~ms}$; TR 2000ms; flip angle $90^{\circ}$ ) during 9:18 min scanning runs incorporating 279 sequential images. Three EPI series were collected from all participants.

\section{Analysis}

Statistical analysis of psychophysical parameters was performed with SPSS V.22.0. Testing included repeated measures analysis of 
variance and $t$ tests. The distributions of cough thresholds and urge-to-cough ratings did not differ significantly to normality (skewness range -0.47 to 1.22 , kurtosis range -1.14 to 1.6 ). Image analysis was performed with the fMRI expert analysis tool (FEAT) V.5.98.22 Regressors representing the timing for each dose of capsaicin and rating events were included in a general linear model that included motion parameters and confound variables to take account of physiological noise according to procedures described previously. ${ }^{19} 23$ These regressors were used to represent the onset, duration and offsets of experimental events (ie, the timing of the saline, low or high dose inhalation blocks and timing of visual cues such as rating and stimulus inhalation cues-refer to online supplementary information for further detail). Contrasts for high and low capsaicin doses were averaged across the three scans for each participant and used in the analysis of group and between-group effects. Group contrasts included high doses for all participants (matched urge-to-cough sensation), and paired doses (matched capsaicin dose). Activation levels of doses matched for urge-to-cough sensation were tested for correlations with $\mathrm{Cu}$ and the frequency of coughing during repeated capsaicin challenges. Significant activations were determined using a single voxel inclusion threshold of $\mathrm{z}>2.3$ and a cluster level threshold of $\mathrm{p}_{\text {corr }}<0.05$ corrected for multiple comparisons based on random field theory using Euler's characteristics. ${ }^{24}$

Further details of the methods are available as online supplementary material.

\section{RESULTS}

\section{Psychophysical responses to capsaicin stimulation}

Behavioural responses to inhalation of nebulised capsaicin showed differences between patients with $\mathrm{CH}(\mathrm{n}=16$, age 43.4 $\pm 15.1,43.8 \%$ male), and the healthy control group ( $\mathrm{n}=16$, age $40.0 \pm 12.0,43.8 \%$ male). The patients with $\mathrm{CH}$ had significantly lower threshold doses of capsaicin to elicit a perceptible urge to cough $(\mathrm{Cu}, \mathrm{t}(30)=2.3, \mathrm{p}=0.026)$, and to evoke two or more coughs $(\mathrm{C} 2, \mathrm{t}(30)=2.2, \mathrm{p}=0.035)$, as well as a lower maximum dose that could be inhaled for $24 \mathrm{~s}$ without coughing $\left(\mathrm{S}_{\max },(\mathrm{t}(30)=2.2, \mathrm{p}=0.038)\right.$ (figure $\left.1 \mathrm{~A}\right)$. Urge-to-cough ratings showed a linear relationship with capsaicin doses $(F(1,30)$ $=171.7, \mathrm{p}<0.001$, partial eta $\left.^{2}=0.851\right)$. However, the linear slope of the urge-to-cough ratings as a function of capsaicin dose did not differ between the two groups $(F(1,30)=0.2$, $\mathrm{p}=0.7)$ nor was there a group difference in mean urge-to-cough ratings $(\mathrm{F}(1,30)=0.5, \mathrm{p}=0.5)$ (figure $1 \mathrm{~B})$. However, there was an overall leftward shift of the stimulus response function for the patients with $\mathrm{CH}$ compared with the controls. Furthermore, the patients coughed more frequently during repeated capsaicin challenges $\quad(t(30)=2.1, \quad p=0.046) \quad$ (figure $1 \mathrm{~B} \quad$ insert). Additionally, patients with $\mathrm{CH}$ were more likely to report the feeling of a spontaneous urge to cough. Thus, during a 12-min restful period, 12 out of 16 patients with $\mathrm{CH}$ reported spontaneous urge to cough (median rating of $2, \mathrm{IQR}=7.8$, range $=0$ $34)$, median of 2 times ( $\mathrm{IQR}=3.8$, range $=0-12$ ) out of 12 occasions they were asked, compared with no reports of spontaneous urge to cough in healthy controls. There were significant differences between controls and patients with $\mathrm{CH}$ with their HARQ total scores $(\mathrm{t}(30)=10.2, \mathrm{p}<0.001)$.

Urge-to-cough ratings collected during functional brain imaging did not differ between the groups when all participants inhaled their individualised $S_{\max }$ dose $(t(30)=0.1, p=0.9)$, despite a systematic difference in stimuli required to achieve a uniform, 'matched urge-to-cough sensation' $(t(30)=2.1$, $p=0.039$ ) (figure 1C). When participants were paired on the
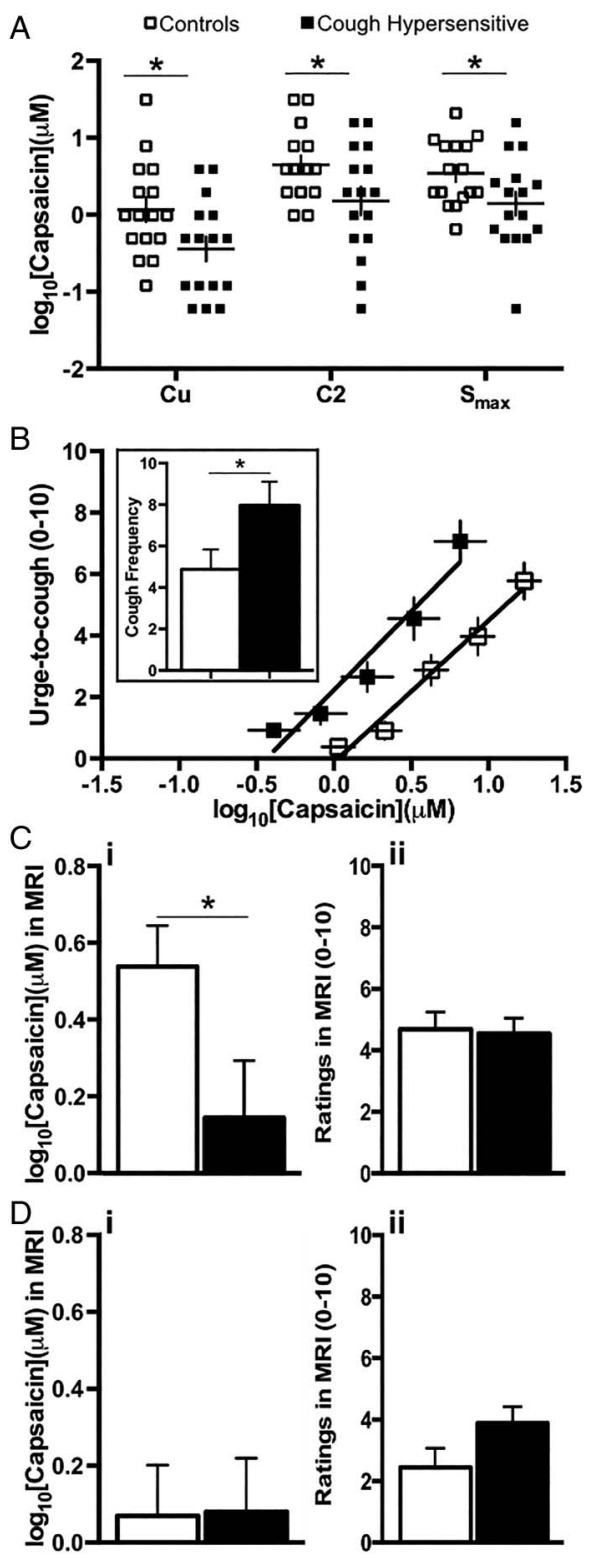

Figure 1 Cough sensitivity was measured in a psychophysical session and during functional brain imaging. (A) Participants inhaled doubling doses of nebulised capsaicin during single breaths. Urge-to-cough ratings (numerical rating scale $0-10$ ) and cough events were recorded. Thresholds were measured for detection of urge to cough (Cu), provocation of two coughs (C2) and maximum suppressible dose during $24 \mathrm{~s}$ of repeated inhalations $\left(S_{\max }\right)$. All three thresholds were significantly decreased in the patients compared with the controls. (B) Relationships between capsaicin doses and urge-to-cough ratings were assessed using repeated inhalations of five capsaicin concentrations at dose increments less than, greater than and corresponding to participants' C2 thresholds. Cough frequency was also recorded (inset). The patients showed a leftward shift of the stimulus/response function and coughed more frequently during challenges. (C) Participants were stimulated with low and high doses of capsaicin during fMRI scanning. 'High' corresponded to participants' $S_{\max }$ and 'Low' was two doses increments below $S_{\max }$. During matched urge-to-cough sensations, despite a significantly lower mean stimulus in the patients compared with controls (i), high doses were associated with similar mean urge-to-cough ratings (ii) in the two groups. (D) Paired comparisons based on capsaicin doses used in the scanner (matched capsaicin dose) were made between age-matched and sex-matched patients and controls. The similar doses (i) were associated with increased urge-to-cough ratings (ii) in the patients. The apparent difference in ratings did not reach significance $(p<0.1)$. ${ }^{*} p<0.05$. 
basis of age and sex and the pair members inhaled the same dose (matched capsaicin dose), there was a discrepancy in mean urge-to-cough ratings, but this difference was not statistically significant $(t(15)=1.7, p=0.1)$ (figure 1D). The urge-to-cough ratings were increased during the scanning session compared with the psychophysical session at comparable high doses for both control and patient groups but not for low doses (highdose mean rating $=4.6 \pm 2.1$ in scanning session and $2.2 \pm 1.8$ in psychophysical session; $\mathrm{F}(1,30)=33.8, \mathrm{p}<0.001$ and low-dose mean rating $=1.5 \pm 1.6$ in scanning session and $1 \pm 1.2$ in psychophysical session; $\mathrm{F}(1,30)=3.4, \mathrm{p}=0.08$ ) observations similar to previous reports from our group. ${ }^{17}$ The absence of a group or interaction effect shows that both groups behaved in the same manner where participants were reporting higher urge-to-cough ratings during repeated inhalations of capsaicin rather than single vital capacity inhalations during the psychophysical session (high dose: $F(1,30)=0.08, p=0.8 ; F(1,30)=0.003, p=1$ and low dose: $F(1,30)=0.9, p=0.4 ; F(1,30)=0.08, p=0.8$ for group and interaction effects, respectively).

\section{Increased levels of capsaicin-inhalation activation in patients with $\mathrm{CH}$ compared with controls}

Patients with $\mathrm{CH}$ and controls showed widely distributed capsaicin-inhalation activations in a network incorporating the mid-cingulate cortex, insula, primary somatosensory and motor cortices, posterior parietal cortices, orbitofrontal cortices, cerebellum, thalamus and brainstem (see online supplementary tables S1, S2 and figure S1). Between-group contrasts showed capsaicin-inhalation activation in the midbrain that was increased in the patients with $\mathrm{CH}$ compared with controls $\left(\mathrm{p}_{\text {corr }}<0.05\right)$. This difference was seen when group contrasts were made for capsaicin-inhalation activations using doses that are matched for urge-to-cough sensations (matched urge-to-cough sensations), and when matched for capsaicin doses (matched capsaicin dose). The increased activation included symmetrical lateral and dorsal regions of the rostral midbrain likely to incorporate the nucleus cuneiformis and the periaqueductal gray (PAG) (figure 2B, C), and the mesial, caudal midbrain encompassing the dorsal raphe (figure 2F, G) (table 1). Mean percentage blood-oxygen-level-dependent (BOLD) signals associated with matched urge-to-cough sensations extracted from the rostral and caudal midbrain regions showed positive increases in the $\mathrm{CH}$ group, whereas controls showed negative mean signal changes (left nucleus cuneiformis: $\mathrm{t}(30)=4.3$, $\mathrm{p}<0.001$; right nucleus cuneiformis: $\mathrm{t}(30)=3.8, \mathrm{p}<0.001$; PAG: $\mathrm{t}(30)=2.9, \mathrm{p}=0.008$ ) (figure $2 \mathrm{D}, \mathrm{H}$ ). Almost all pairs of BOLD signal changes for matched capsaicin doses were increased in the patients with $\mathrm{CH}$ compared with the controls in the midbrain regions (left nucleus cuneiformis: $t(15)=4.5$, $\mathrm{p}<0.001$; right nucleus cuneiformis: $\mathrm{t}(15)=3.4, \mathrm{p}=0.004$; PAG $\mathrm{t}(15)=3.0, \quad \mathrm{p}=0.009$ ) (figure 2E, I). Levels of capsaicin-inhalation activation during matched urge-to-cough sensation in the nucleus cuneiformis on the left $(x=-14, y=$ $-26, \mathrm{z}=-14, \mathrm{z}$ score $=3.92)$ and right $(\mathrm{x}=14, \mathrm{y}=-26, \mathrm{z}=-16$,
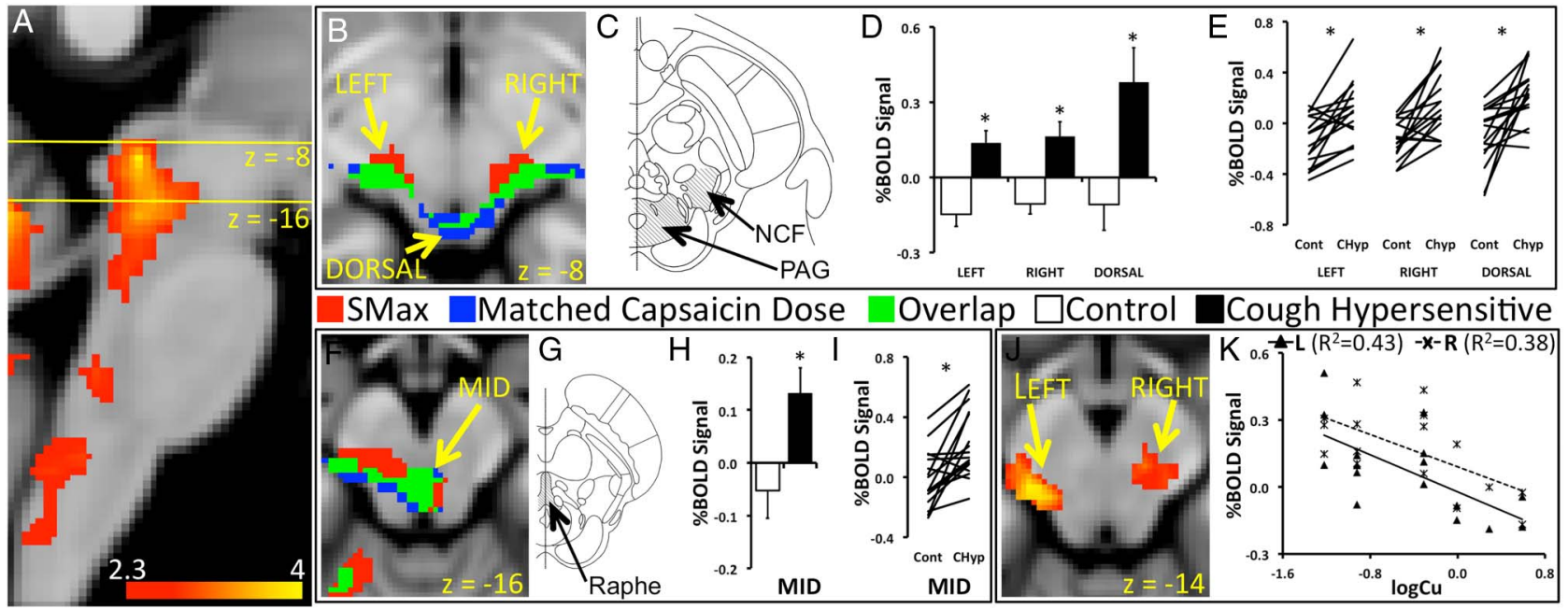

Figure 2 Participants inhaled capsaicin during acquisition of functional brain images. (A) Patients showed capsaicin-inhalation activation in the brainstem. The yellow lines in the sagittal section of the brainstem indicate the location of axial cross-sections shown in (B) and (F). (B) Two types of contrasts of capsaicin-inhalation responses were made between patients and controls. One contrast involved between-group differences of activations associated with $S_{\max }$ doses of capsaicin (matched urge-to-cough sensations). The second contrast used responses to like-doses among age and sex-matched pairs of patients and controls (matched capsaicin dose). Regions in the left, right and dorsal midbrain showed increased capsaicin-inhalation activation in patients compared with controls in response to matched urge-to-cough sensation (red) and matched capsaicin dose (blue). The regions of activation of the two type of contrast overlapped (green). (C) The increased capsaicin inhalation in the patients was in regions likely to incorporate the nucleus cuneiformis and the periaqueductal gray (PAG). (D) Blood-oxygen-level-dependent (BOLD) signal changes associated with matched urge-to-cough sensations extracted from the three regions in the midbrain showed significantly increased levels in the patients, and had mean negative values in the healthy cohort. (E) BOLD signals associated with matched capsaicin dose showed increased levels in the patients (CHyp) compared with controls (Cont) for the majority of pairs in the three regions of the midbrain. (F) Increased capsaicin-inhalation activation in the patients compared with controls was also seen in the mid region of the brainstem (MID) at $z=-16$. (G) The MID region was likely to encompass the PAG and the dorsal raphe of the midbrain (Raphe). (H) BOLD signal changes in the MID region showed divergent increases and decreases in the patients and controls. (I) Matched capsaicin dose elicited increased BOLD signal changes for most pairs of patients versus controls in the MID region. $(\mathrm{J})$ Left and right midbrain regions showed correlations between levels of activation associated with matched urge-to-cough sensations and cough sensitivity represented by the $\mathrm{Cu}$ threshold $(\log \mathrm{Cu})$ in the patients. $(\mathrm{K})$ BOLD signal changes associated with matched urge-to-cough sensations were extracted from the left and right midbrain to demonstrate the nature of the relationship with cough hypersensitivity. The slopes of the lines indicate that the patients with the greatest levels of sensitivity (low Cu thresholds) showed the highest levels of BOLD signal change in the left and right midbrain. ${ }^{*} \mathrm{p}<0.05$. 
Table 1 Capsaicin-inhalation activation, patients with cough hypersensitivity $>$ controls

\begin{tabular}{|c|c|c|c|c|c|c|c|c|}
\hline \multirow[b]{3}{*}{ Region } & \multicolumn{4}{|c|}{$\begin{array}{l}\text { Matched urge-to-cough } \\
\text { sensation }\end{array}$} & \multicolumn{4}{|c|}{ Matched capsaicin dose } \\
\hline & \multicolumn{3}{|c|}{$\begin{array}{l}\text { Peak voxel } \\
\text { coordinate }\end{array}$} & \multirow{2}{*}{$\begin{array}{l}\text { z } \\
\text { score }\end{array}$} & \multicolumn{3}{|c|}{$\begin{array}{l}\text { Peak voxel } \\
\text { coordinate }\end{array}$} & \multirow{2}{*}{$\begin{array}{l}\text { z } \\
\text { score }\end{array}$} \\
\hline & $\mathbf{x}$ & y & $\mathbf{z}$ & & $\mathbf{x}$ & $y$ & $\mathbf{z}$ & \\
\hline $\begin{array}{l}\text { N. Cuneiformis } \\
\text { (L) }\end{array}$ & -14 & -24 & -6 & 3.31 & -14 & -26 & -8 & 3.62 \\
\hline $\begin{array}{l}\text { N. Cuneiformis } \\
\text { (R) }\end{array}$ & 16 & -24 & -6 & 3.28 & 14 & -24 & -8 & 3.23 \\
\hline PAG & -2 & -34 & -10 & 2.99 & 2 & -34 & -8 & 2.73 \\
\hline Dorsal Raphe & 2 & -28 & -16 & 2.91 & 0 & -28 & -16 & 3.26 \\
\hline
\end{tabular}

$\mathrm{z}$ score $=3.0)$ correlated with $\mathrm{Cu}$ thresholds in the patients $\left(\mathrm{p}_{\text {corr }}<0.05\right)$ (figure $\left.2 \mathrm{~J}\right)$. Patients with the highest levels of sensitivity (low $\mathrm{Cu}$ thresholds) had the highest levels of BOLD signal change in the lateral midbrain (left $\mathrm{R}^{2}=0.4, \mathrm{p}=0.005$; right $\mathrm{R}^{2}=0.4, \mathrm{p}=0.011$ ) (figure $2 \mathrm{~K}$ ).

\section{Increased levels of capsaicin-inhalation activation in controls compared with patients with $\mathrm{CH}$}

Controls showed an increase in capsaicin-inhalation activation in the dorsomedial prefrontal cortex (DMPFC) $(x=-2, y=32$, $\mathrm{z}=36, \mathrm{z}$ score $=3.5$ ) and anterior mid-cingulate cortices (aMCC) $(x=-2, y=18, z=24, z$ score $=3.4)$ compared with patients with $\mathrm{CH}$ in response to matched urge-to-cough sensations ( $\mathrm{p}_{\text {corr- }}$ $<0.05$ ) (figure $3 \mathrm{~A}$ ). These regions have previously been implicated in cough suppression, ${ }^{19}$ and the doses used for matched urge-to-cough sensations were tailored for each participant to be the maximum that could be inhaled for $24 \mathrm{~s}$ without coughing. The BOLD signal changes extracted from the DMPFC in the controls showed increases during the high capsaicin dose when cough suppression was compliant with the protocol, whereas signal changes were approaching zero in response to lower capsaicin doses when the need for suppression was unlikely $(\mathrm{t}(15)=3.0, \mathrm{p}=0.009)$ (figure $3 \mathrm{~B})$. In contradistinction to the controls, BOLD signal changes in the DMPFC of the patients with $\mathrm{CH}$ were negative during inhalation of both high and low doses of capsaicin $(t(15)=0.2, p=0.86)$.

The network implicated in cough suppression extends beyond the medial prefrontal cortex to include other prefrontal and paralimbic regions. While between-group differences in the cough-suppression network were confined to the DMPFC and aMCC, other constituent regions showed an association between activation levels during matched urge-to-cough sensations and the frequency of coughing events recorded during the psychophysical session (figure $3 \mathrm{C}$ and table 2). This relationship was seen exclusively in the patients with $\mathrm{CH}\left(\mathrm{p}_{\mathrm{corr}}<0.05\right)$ and included the right anterior insula and inferior frontal gyrus. BOLD signal changes from anterior insula $\left(R^{2}=0.6, p<0.001\right)$ and inferior frontal gyrus $\left(\mathrm{R}^{2}=0.6, \mathrm{p}<0.001\right)$ during matched urge-to-cough sensations were lowest in the patients that coughed with the greatest frequency during repeated capsaicin challenges in the psychophysical session (figure 3D).

\section{DISCUSSION}

The results of our study identified two outcomes. First, patients with $\mathrm{CH}$ displayed increased neural activity, correlating with
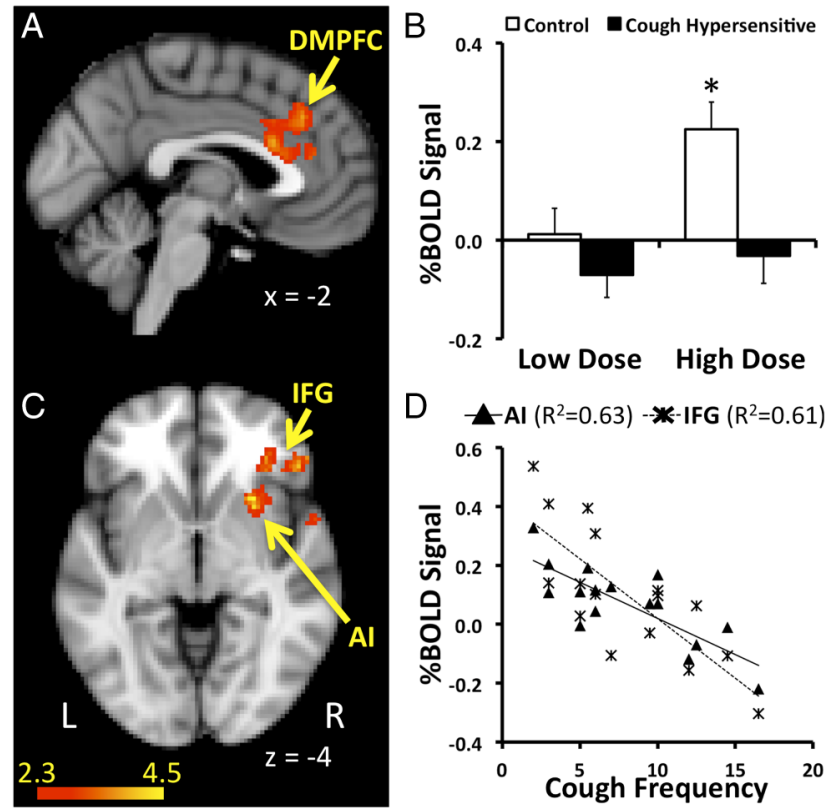

Figure 3 (A) Between-group contrasts of responses to the maximum suppressible dose ( $S_{\max }$, matched urge-to-cough sensations) revealed regions in the dorsomedial prefrontal cortex (DMPFC) and neighbouring anterior cingulate cortex where healthy controls showed significantly increased levels of capsaicin-inhalation activation compared with patients. (B) The pattern of blood-oxygen-level-dependent (BOLD) signal responses of the DMPFC in the controls was consistent with a cough-suppression function. Positive signal changes occurred during a high dose likely to elicit coughing without active suppression, while low doses associated with minor levels of urge to cough and unlikely to elicit coughing were associated with minimal signal change. On average, the patients showed negative BOLD signal changes in the DMPFC in response to the two doses. (C) Levels of capsaicin-inhalation activation in the patients correlated with the frequency of coughing elicited during repeated challenges in the psychophysical session. These correlations included two regions implicated in cough suppression, the right anterior insula (Al) and right inferior frontal gyrus (IFG). (D) Patients that coughed with the greatest frequency during capsaicin challenge had the lowest levels of BOLD signal change during capsaicin inhalation in the Al and IFG. ${ }^{*} p<0.05$.

measures of sensory sensitivity, in midbrain regions not activated in controls undergoing the same experimental challenges. Second, patients with $\mathrm{CH}$ displayed reduced activity in a central network involved in cough suppression, ${ }^{16} 1925$ and this related to their inability to control coughing. Collectively, these data suggest a central contribution to cough hypersensitivity that

Table 2 Regions of capsaicin inhalation (matched urge-to-cough sensations) that correlated with cough frequency in the patients with cough hypersensitivity

\begin{tabular}{lllll}
\hline & \multicolumn{3}{l}{ Peak voxel coordinate } & \\
\cline { 2 - 4 } Region & $\mathbf{x}$ & $\mathbf{y}$ & $\mathbf{z}$ & z score \\
\hline Inferior frontal gyrus & 36 & 36 & -8 & 4.71 \\
Anterior insula & 28 & 16 & -4 & 4.61 \\
Frontal operculum & 46 & 14 & -2 & 4.77 \\
Sup. temporal gyrus & 62 & 6 & -10 & 4.10 \\
Putamen & 28 & 14 & 0 & 4.37 \\
Caudate & 18 & 14 & 6 & 3.46 \\
\hline
\end{tabular}


may lead to excessive coughing due to altered interoceptive processing and diminished capacity to engage central cough control mechanisms. ${ }^{26}$

\section{Brain activity associated with airways irritation}

Cough occurs within a functional spectrum ranging from purely reflex to purely voluntary, and is almost always accompanied by perceivable sensations of unpleasantness that drive a desire (or urge) to cough. ${ }^{1}$ This complexity is reflected in the central pathways that regulate cough, which have been described using functional brain imaging in healthy humans. The transient sense of laryngeal irritation that precedes the need for coughing following inhalation of capsaicin is associated with activations in a distributed brain network that presumably encode sensory dimensions of the experience and/or accompanying motorrelated responses. ${ }^{16-20}$ This includes activity in the somatosensory, motor, premotor, prefrontal, limbic and paralimbic cortices, in which distinct patterns of activity relate to different aspects of sensory discrimination and/or motor control. For example, neural activity in the primary sensory cortex correlates with an individual's perception of urge-to-cough intensity, ${ }^{16}$ while activity in the inferior frontal gyrus, DMPFC, anterior insula cortex and anterior mid-cingulate cortex relates to the motor task of cough suppression. ${ }^{17} 19$ Other regional responses appear important for encoding stimulus intensity, ${ }^{16}$ voluntary cough $^{19} 27$ and for placebo-evoked suppression of cough. ${ }^{18}$ In the present study, the broader network of brain responses associated with capsaicin inhalation in healthy participants mirrored what we have reported previously. ${ }^{16} 20$

\section{Elevated midbrain activity in patients with $\mathrm{CH}$}

Patients with $\mathrm{CH}$ had increased sensitivity to inhaled capsaicin compared with controls accompanied by between-group differences in BOLD signal response in the midbrain, localised bilaterally in the nucleus cuneiformis and extending into the midline PAG and dorsal raphe nucleus. On average, the healthy control group demonstrated no activation above baseline in these midbrain regions and the resultant between-group differences were evident in both comparisons of the same stimulus intensities and the same urge-to-cough experiences. We further noted a significant relationship between the percentage BOLD signal change in the nucleus cuneiformis and the patients' urge-to-cough sensitivity scores $(\mathrm{Cu})$. Comparable midbrain activity has been reported during somatic and visceral pain ${ }^{28}$ and in subjects following induction of pain hypersensitivity. ${ }^{14}$ Thus, subjects treated on the lower leg with a combination of heat and capsaicin experience a transient hyperalgesia to subsequent mechanical stimulation of limb, and this is associated with increased neural activity in the midbrain nucleus cuneiformis. Collectively, these data are indicative of the midbrain playing a central role in the development of nociceptive sensitisation in both chronic cough and pain, adding to the growing evidence that cough and pain share common mechanistic processes. 3101129

The nucleus cuneiformis, PAG and raphe are all component nuclei of a descending pain modulatory system, the activation of which can both enhance and inhibit nociceptive transmission at the level of the spinal cord. ${ }^{13}{ }^{30}$ This bimodal control occurs via distinct excitatory (on cells) and inhibitory (off cells) neurons residing in the rostral ventromedial medulla, which receive input commands from both the midbrain and the spinal cord, and in turn regulate output to the dorsal horn of the spinal cord where incoming nociceptive signals are first integrated. ${ }^{30}$ In animal studies, alterations in descending control can induce hyperalgesia in the absence of peripheral injury. ${ }^{31} 32$ Neural circuit tracing studies provide an anatomical framework for airway sensory pathways innervating the descending pain modulatory system ${ }^{25} 33$ which in turn regulate medullary brainstem regions that process airway sensory inputs. ${ }^{34}$ Thus, patients with $\mathrm{CH}$, like those with chronic pain, may develop hypersensitivity due to changes in descending control. Whether the altered midbrain activity in patients with $\mathrm{CH}$ manifests only as a change in coughing control or if it also induces a more generalised change in descending somatosensory control is presently unknown.

\section{Diminished activity in cough-suppression networks in chronic cough}

We have previously defined a network of brain activity involving the right inferior frontal gyrus, right anterior insula cortex, DMPFC, anterior mid-cingulate cortex and supplementary motor area, ${ }^{16} 171925$ requisite for the active suppression of evoked coughing. This network closely resembles that engaged during other types of motor suppression (eg, the go/no-go response inhibition task). ${ }^{35}$ In the present study, patients with $\mathrm{CH}$ responded with significantly more coughs than did controls during capsaicin challenges that evoked comparable urge-to-cough sensations, perhaps indicative of diminished central suppression contributing to heightened cough motor output. Indeed, patients with $\mathrm{CH}$ demonstrated less activation in the DMPFC and aMCC, areas activated at high stimulus intensities in healthy participants consistent with their role in

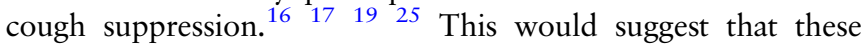
patients have reduced capacity to actively suppress coughing. The correlation between cough frequency and diminished cough-suppression network activity is also compelling evidence in this regard. Thus, we noted that the patients who exhibited the most difficulty in controlling their cough (ie, those with the highest cough frequency) displayed the least activity in their right inferior frontal gyrus and the right anterior insula.

\section{Implications and conclusions}

The emerging view of chronic cough is that of a sensory neuropathy, akin to the inflammatory and neuropathic processes that lead to development of chronic pain. ${ }^{3}$ Our data support this notion showing that patients with $\mathrm{CH}$ have altered brain activity that reflects the clinical presentation of their disorder. Of note, the central manifestations reported here probably reflect common central mechanisms underpinning $\mathrm{CH}$ disorder as the present study enlisted a group of patients with $\mathrm{CH}$ with heterogeneous underlying clinical presentations. The similarity in the midbrain response that develops in cough and pain hypersensitivity argues that these distinct clinical entities may be linked by an extraordinarily common central neurological basis. Whether the diminished capacity to engage cough suppression develops secondary to altered sensory processing or, alternatively, is independent or even inherent in patients with chronic cough is unclear. Our data also provide new insights into therapeutic relief of chronic cough. Indeed, it is intriguing that speech therapy techniques have proven effective for controlling cough in some patients, ${ }^{36}$ which may potentially relate to improvements in central cough-suppression network activity, although this awaits further study. The current data also provide a neurobiological basis for why neuroactive drugs such as gabapentin may provide cough relief ${ }^{11}$ and further encourage the exploration of the CNS as a site for intervention to improve troublesome cough. Nevertheless, the stimulus employed, capsaicin, is a selective activator of only one subset of chemically sensitive cough evoking sensory nerves (namely C-fibres) and therefore it does not provide insight into cough evoked via mechanoreceptor 
pathways which may be important in disease. ${ }^{37}$ Thus, whether there are additional central neural correlates of dysfunctional cough remains to be determined. Furthermore, although the present data demonstrate central neural changes in patients with $\mathrm{CH}$, we do not know the relative dependence of these central changes on enhanced peripheral sensory activation that may be upregulated in patients with $\mathrm{CH}$.

Acknowledgements We acknowledge the technical expertise provided by Michael Kean of the Children's MRI Centre (Melbourne, Australia).

Contributors AA, SBM and MJF were involved in planning, conducting and reporting of the work described in the article. DS, MM and LI were involved in planning and recruitment of participants.

Funding This research was supported by grants to SBM and MJF from the National Health and Medical Research Council (NHMRC) of Australia (1042528, 1025589, 1078943).

Competing interests None declared.

Ethics approval Melbourne Health Human Research Ethics Committee.

Provenance and peer review Not commissioned; externally peer reviewed.

Data sharing statement Unpublished data from the study are only available to authors on this manuscript.

\section{REFERENCES}

1 Ando A, Farrell MJ, Mazzone SB. Cough-related neural processing in the brain: a roadmap for cough dysfunction? Neurosci Biobehav Rev 2014;47:457-68.

2 Farrell MJ, Mazzone SB. Sensations and regional brain responses evoked by tussive stimulation of the airways. Respir Physiol Neurobiol 2014;204:58-63.

3 Chung KF, McGarvey L, Mazzone SB. Chronic cough as a neuropathic disorder. Lancet Respir Med 2013;1:414-22.

4 Hilton E, Marsden P, Thurston A, et al. Clinical features of the urge-to-cough in patients with chronic cough. Respir Med 2015;109:701-7.

5 McGarvey L, McKeagney P, Polley L, et al. Are there clinical features of a sensitized cough reflex? Pulm Pharmacol Ther 2009;22:59-64.

6 Morice $\mathrm{AH}$, Millqvist E, Belvisi MG, et al. Expert opinion on the cough hypersensitivity syndrome in respiratory medicine. Eur Respir J 2014;44:1132-48.

7 Song WJ, Chang YS, Morice AH. Changing the paradigm for cough: does 'cough hypersensitivity' aid our understanding? Asia Pac Allergy 2014;4:3-13.

8 McGarvey L. The difficult-to-treat, therapy-resistant cough: why are current cough treatments not working and what can we do? Pulm Pharmacol Ther 2013;26:528-31.

9 Pratter MR. Unexplained (idiopathic) cough: ACCP evidence-based clinical practice guidelines. Chest 2006;129(1 Suppl):220S-21S.

10 Mazzone SB, McGovern AE, Koo K, et al. Mapping supramedullary pathways involved in cough using functional brain imaging: comparison with pain. Pulm Pharmacol Ther 2009;22:90-6.

11 Ryan NM, Birring SS, Gibson PG. Gabapentin for refractory chronic cough: a randomised, double-blind, placebo-controlled trial. Lancet 2012;380:1583-9.

12 Scholz J, Woolf CJ. The neuropathic pain triad: neurons, immune cells and glia. Nat Neurosci 2007;10:1361-8.

13 Tracey I, Mantyh PW. The cerebral signature for pain perception and its modulation. Neuron 2007:55:377-91.

14 Zambreanu L, Wise RG, Brooks JC, et al. A role for the brainstem in central sensitisation in humans. Evidence from functional magnetic resonance imaging Pain 2005; 114:397-407.
15 Undem BJ, Taylor-Clark T. Mechanisms underlying the neuronal-based symptoms of allergy. J Allergy Clin Immunol 2014;133:1521-34.

16 Farrell MJ, Cole LJ, Chiapoco D, et al. Neural correlates coding stimulus level and perception of capsaicin-evoked urge-to-cough in humans. Neurolmage 2012;61:1324-35.

17 Farrell MJ, Koch $\mathrm{S}$, Ando $\mathrm{A}$, et al. Functionally connected brain regions in the network activated during capsaicin inhalation. Hum Brain Mapp 2014;35: 5341-55.

18 Leech J, Mazzone SB, Farrell MJ. Brain activity associated with placebo suppression of the urge-to-cough in humans. Am J Respir Crit Care Med 2013;188:1069-75.

19 Mazzone SB, Cole LJ, Ando A, et al. Investigation of the neural control of cough and cough suppression in humans using functional brain imaging. J Neurosci 2011:31:2948-58.

20 Mazzone SB, McLennan L, McGovern AE, et al. Representation of capsaicin-evoked urge-to-cough in the human brain using functional magnetic resonance imaging. Am J Respir Crit Care Med 2007;176:327-32.

21 Morice $\mathrm{AH}$, Faruqi $\mathrm{S}$, Wright $\mathrm{CE}$, et al. Cough hypersensitivity syndrome: a distinct clinical entity. Lung 2011;189:73-9.

22 Jenkinson M, Beckmann CF, Behrens TE, et al. FSL. Neurolmage 2012;62:782-90.

23 Birn RM, Murphy K, Handwerker DA, et al. fMRI in the presence of task-correlated breathing variations. Neurolmage 2009;47:1092-104.

24 Worsley KJ, Evans AC, Marrett $S$, et al. A three-dimensional statistical analysis for CBF activation studies in human brain. I Cereb Blood Flow Metab 1992:12:900-18

25 Mazzone SB, McGovern AE, Farrell MJ. Endogenous central suppressive mechanisms regulating cough as potential targets for novel antitussive therapies. Curr Opin Pharmacol 2015;22:1-8.

26 Barrett LF, Simmons WK. Interoceptive predictions in the brain. Nat Rev Neurosci 2015; 16:419-29

27 Simonyan K, Saad ZS, Loucks TM, et al. Functional neuroanatomy of human voluntary cough and sniff production. Neurolmage 2007;37:401-9.

28 Dunckley $\mathrm{P}$, Wise RG, Fairhurst $\mathrm{M}$, et al. A comparison of visceral and somatic pain processing in the human brainstem using functional magnetic resonance imaging. J Neurosci 2005;25:7333-41.

29 O'Neill J, McMahon SB, Undem BJ. Chronic cough and pain: Janus faces in sensory neurobiology? Pulm Pharmacol Ther 2013;26:476-85.

30 Lau BK, Vaughan CW. Descending modulation of pain: the GABA disinhibition hypothesis of analgesia. Curr Opin Neurobiol 2014;29:159-64.

31 Cai YQ, Wang W, Hou YY, et al. Optogenetic activation of brainstem serotonergic neurons induces persistent pain sensitization. Mol Pain 2014;10:70.

32 Guo W, Miyoshi K, Dubner R, et al. Spinal 5-HT3 receptors mediate descending facilitation and contribute to behavioral hypersensitivity via a reciprocal neuron-glial signaling cascade. Mol Pain 2014;10:35.

33 McGovern AE, Driessen AK, Simmons DG, et al. Distinct brainstem and forebrain circuits receiving tracheal sensory neuron inputs revealed using a novel conditional anterograde transsynaptic viral tracing system. J Neurosci 2015;35:7041-55.

34 Sessle BJ, Ball GJ, Lucier GE. Suppressive influences from periaqueductal gray and nucleus raphe magnus on respiration and related reflex activities and on solitary tract neurons, and effect of naloxone. Brain Res 1981;216:145-61.

35 Aron AR, Robbins TW, Poldrack RA. Inhibition and the right inferior frontal cortex: one decade on. Trends Cogn Sci (Regul Ed) 2014;18:177-85.

36 Vertigan AE, Theodoros DG, Gibson PG, et al. Efficacy of speech pathology management for chronic cough: a randomised placebo controlled trial of treatment efficacy. Thorax 2006:61:1065-9.

37 Canning BJ, Mazzone SB, Meeker SN, et al. Identification of the tracheal and laryngeal afferent neurons mediating cough in anaesthetized Guinea-pigs. J Physiol (Lond) 2004;557(Pt 2):543-58. 